\title{
Longer orthodontic treatment may result in greater external apical root resorption
}

\author{
What are the treatment-related aetiological factors of external apical root \\ resorption of the maxillary incisor?
}

\begin{abstract}
Segal GR, Schiffman PH, Tuncay OC. Meta analysis of the treatment-related factors of external apical root resorption. Orthod Craniofac Res 2004; 7:71-78.
\end{abstract}

Data sources Medline was the primary data source, with references of identified papers then being reviewed for additional studies.

Study selection Studies were included if they were clinical trials, in English, of samples of greater than 10 subjects who had undergone fixed appliance orthodontic treatment and who had both pre- and postoperative $\mathrm{X}$-rays available. A measurement of external apical root resorption (EARR) in maxillary incisors was essential as was a measure of displacement with the apex of the root as reference.

Data extraction and synthesis Variables were coded and articles graded by three independent investigators who subsequently negotiated final coding and assessed the methodological soundness of each study. A cumulative 'meta-analysis factor' was computed for each article. Articles were evaluated on study design; population sample; treatment assignment; documentation of statistics; the accuracy of root resorption measurement and apical displacement of incisor roots. A funnel plot analysis did not identify publication bias.

Results Eight articles were included in the statistical analysis. Two did not contain data for mean apical displacement and therefore correlations between mean EARR and mean apical displacement could not be calculated for them. The mean meta-analysis factor was 39 (range, 1278). Mean root resorption was $1.421 \pm 0.448 \mathrm{~mm}(n=8)$, and mean apical displacement was $2.382 \pm 0.756 \mathrm{~mm}$. The weighted correlation coefficient between mean root resorption and apical displacement was 0.822 and that between mean root resorption and treatment duration was 0.852 .

Conclusions This analysis suggests that treatment-related root resorption is correlated with the distance the apex moves and the length of time the treatment took.
Address for correspondence: G Ross Segal, 1518 Walnut Street, Suite \#500, Philadelphia PA 19102, USA.

\section{Commentary}

Orthodontic treatment has been associated with apical root resorption for many years, but a consensus on treatment-related causes of apical resorption cannot be found in the literature. Factors often quoted in orthodontic texts are: magnitude of force application, duration of treatment, morphology of teeth and the nature of the adjacent bone. Radiographic evidence of pretreatment root resorption is also felt to be a significant factor. The problem is that, when reading the literature, reports that support or refute the above claims can be found in equal numbers.

The best way, from a scientific point of view, would obviously be to investigate all of the above factors with a prospective randomised clinical trial, but of course it would be unethical to do so. Indeed, now that the use of postorthodontic treatment radiographs is not considered a routine requirement, retrospective studies will also be more difficult to carry out.

This paper investigated treatment-related aetiological factors of EARR through meta-analytic assessment. Meta-analysis is the statistical analysis of a sample of analyses taken from individual studies for the purpose of integrating findings to produce a greater weight of evidence. The authors found around 150 studies investigating EARR in relation to orthodontics, but only nine met their inclusion and exclusion criteria as detailed in the above summary.

Their results support the anecdotal evidence believed by many clinical orthodontists, that the degree of root resorption is correlated with the distance the apex of an incisor moves and the length of time of the orthodontic treatment. My only criticism of the paper is the decision to exclude patients and studies with a history of prior root resorption. Although I can understand the reason for this, as a clinical orthodontist it would be very useful to have some evidence to support or refute the claim that such patients would be more prone to resorption during orthodontic treatment or indeed whether it is appropriate to treat such cases.

This study highlights two important issues. First is the fact that fewer than $10 \%$ of published studies could be included in what I thought were fairly generous inclusion criteria. The need for consistent research methods in this important area of clinical dentistry is once again highlighted. Secondly, factors such as magnitude of the force applied and morphology of teeth, factors that are anecdotally associated with root resorption, cannot be quantified.

\section{Practice point}

- Patients who require prolonged orthodontic treatment or treatment involving a significant degree of tooth movement should be warned about resorption prior to orthodontic treatment.

\section{Nigel Fox}

James Cook University Hospital, Middlesbrough, Cleveland, UK

Evidence-Based Dentistry (2005) 6, 21.

doi:10.1038/sj.ebd.6400304 